\title{
Non-passerine birds from Laguna Melincué Ramsar Site, Santa Fe province, Argentina
}

\author{
Marcelo Romano $^{1}$, Ignacio Martín Barberis ${ }^{2 *}$, Marcelo Luppi $^{3}$ and Fernando Pagano ${ }^{4}$ \\ 1 Centro de Investigaciones en Biodiversidad y Ambiente (ECOSUR). Pasaje Sunchales 329. CP2000. Rosario, Santa Fe, Argentina \\ 2 Instituto de Investigaciones en Ciencias Agrarias, Consejo Nacional de Investigaciones Científicas y Técnicas and Universidad Nacional \\ de Rosario, Facultad de Ciencias Agrarias. Campo Experimental Villarino, C.C. 14. S2125ZAA, Zavalla, Santa Fe, Argentina \\ 3 Comuna de Melincué, San Lorenzo 579, CP2728, Melincué, Santa Fe, Argentina \\ 4 Universidad Nacional de Rosario, Facultad de Ciencias Veterinarias. Bvd. Ovidio Lagos y Ruta 33, C.C. 166, S2170HGJ Casilda, Santa Fe, \\ Argentina \\ * Corresponding author. E-mail: ignaciobarberis@yahoo.com
}

\begin{abstract}
We present a waterbird inventory of the Laguna Melincué Ramsar Site in southern Santa Fe province, Argentina. We record 109 species from 28 families of non-passerine birds, including the 14 Nearctic species, four southern austral migrant species, three northern austral migrants, 17 partial migrants, and two altitudinal migrants. The eight most abundant species belong to different families and trophic groups. Thirty-six species were observed nesting or rearing chicks. This baseline knowledge of non-passerine birds will be useful to assist future conservation studies in this highly threatened area.
\end{abstract}

Key words: agroecosystems; Pampas; waterbirds; wetlands; Laguna Melincué Ramsar Site

\section{INTRODUCTION}

The grassland vegetation of the Argentine Pampas has been completely modified over the last century by intensive crop production, as well as for pastures for cattle raising (Vega et al. 2009; Viglizzo et al. 2011). Nowadays, the modified landscapes of these extensive plains are mainly composed of a few crop species (e.g., soybean, maize and wheat) and pastures (alfalfa and ryegrass). There are, however, many scattered wetlands within this homogeneous and low-diversity matrix of agroecosystems. These wetlands are known for providing several ecosystem services (Barral and Maceira 2012; Laterra et al. 2012), and some of them are key habitats for many resident and migratory waterbirds (Romano et al. 2005; de la Peña 2010; Brandolin et al. 2011). Many of these wetlands are subject to several pressures and threats, such as agriculture, livestock raising, water management and hunting (Marconi and Sureda 2008; Brandolin et al. 2013).
Despite their conservation value, most of these wetlands have no legal protection (Canevari et al. 1998). One exception is Laguna Melincué, a large wetland recognized as an Important Bird Area (Di Giacomo et al. 2005), which was declared Ramsar site in 2008 due to its importance for waterbirds (The Ramsar Convention on Wetlands 2013). We have been conducting nonpasserine waterbird surveys at Laguna Melincué for more than 20 years. In this work, we update our own and others' previous published waterbird and landbird data (Romano et al. 2005; Saggesse et al. 2007; Giraudo et al. 2008), including new records of non-passerine species taken during the last 12 years.

\section{MATERIAL AND METHODS}

Laguna Melincué is located in Santa Fe province, Argentina $\left(33^{\circ} 43^{\prime} \mathrm{S}, 061^{\circ} 28^{\prime} \mathrm{W}, 84 \mathrm{~m}\right.$ above sea level; Figure 1). Its watershed comprises $678 \mathrm{~km}^{2}$ and is the collection basin of several wet meadows. This shallow lake has a maximum depth about 6-7 $\mathrm{m}$ and a highly variable surface area ranging from $48-145 \mathrm{~km}^{2}$. The water is moderately saline (about $3-6 \mathrm{~g} / \mathrm{dm}^{3}$ of total dissolved solids), with high $\mathrm{pH}$ (range 9-11) and low water transparency (about $0.15 \mathrm{~m}$ with a Secchi disk) (Romano et al. 2005, 2008). Water level fluctuations expose extensive mud flats and the surrounding vegetation is mainly composed by halophilous prairies dominated by Distichlis spicata and Paspalum vaginatum (Romano et al. 2005). Grasslands surrounding these wetlands are mainly used for cattle raising.

We have conducted systematic annual waterbird surveys at Laguna Melincué Ramsar Site for more than 20 years (1992-2014). Each year, we identified and counted waterbirds along several transects using binoculars (10x) and spotting scopes (15/45x and 22/67x). The 


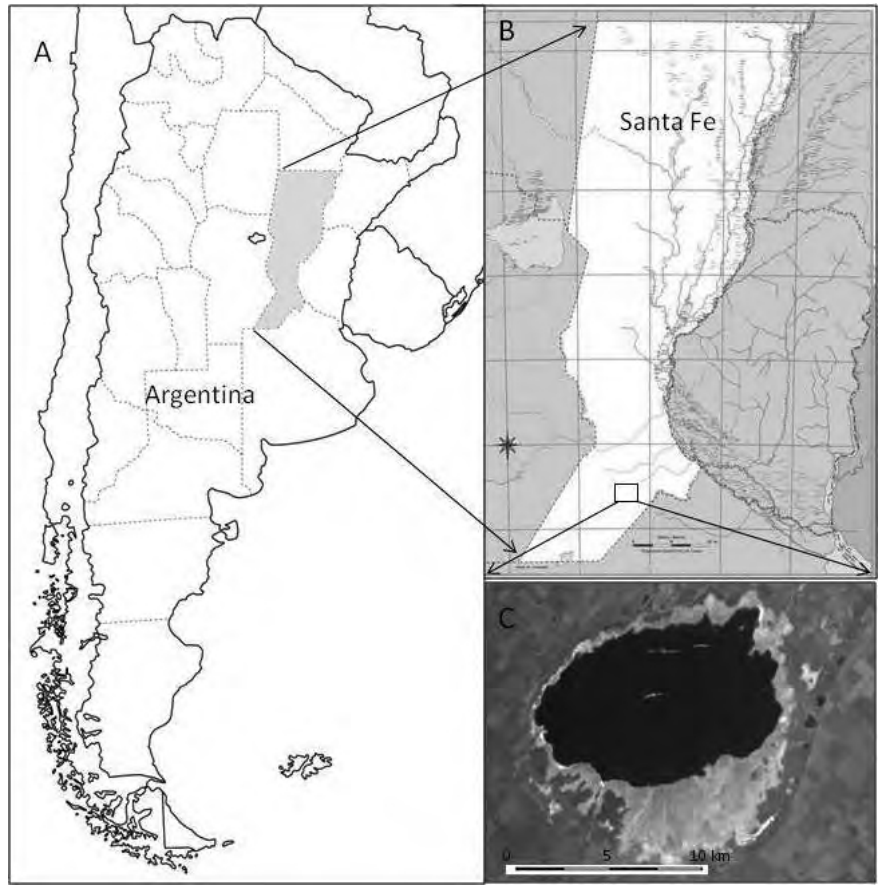

Figure 1. Location of the study site in Laguna Melincué Ramsar Site, Santa Fe province, Argentina.

information of the first 10 years (1992-2002) has been published elsewhere (Romano et al. 2005). Now, we add some new records based on similar waterbird surveys carried out during the last 12 years (2003-2014). Raptors were recorded during a study evaluating the presence of anthrax spores in their feces (Saggesse et al. 2007). Finally, other landbird species were recorded while doing the systematic waterbird censuses, as well as by personal records taken by one of us, M. Luppi, while doing his duty as an official fauna keeper at Melincué. During all these surveys we recorded nesting activities or the presence of juveniles.

Species were classified according to their relative abundances for the whole study period as very abundant (>10,000 observations), abundant (1,000-10,000 observations), scarce (100-1,000 observations), and rare (<100 observations) (Brandolin et al. 2011). We followed Fandiño and Giraudo (2010) and Brandolin et al. (2011) to classify species as 'residents' or 'migrants'. The latter were divided in 'Nearctic migrants' (i.e., species that nest in the Nearctic and then migrate to the south being recorded in the area in spring and summer), 'northern austral migrants' (i.e., species that nest in the area in spring and summer and then migrate to northern areas), 'southern austral migrants' (i.e., species that nest in southern Argentina in spring and summer and disperse to the study area in autumn and winter), 'Altitudinal Migrants' (i.e., species that nest in the High Andes in summer and migrate to the area in winter) and 'Partial Migrants' (i.e., species that move from the study area to other areas, but without any clear pattern).

\section{RESULTS}

We recorded the presence of 109 species from 28 families of non-passerine birds (Table 1). The more diverse families were Anatidae (16 species), Scolopacidae (13 species), Accipitridae (9 species) and Ardeidae (8 species). Most bird species are resident in the area. However, we recorded 14 Nearctic species: Buteo swainsoni Bonaparte, 1838, Pluvialis dominica (Statius Muller, 1776), Limosa haemastica (Linnaeus, 1758), Bartramia longicauda (Bechstein, 1812), Tringa melanoleuca (Gmelin, 1789), Tringa flavipes (Gmelin, 1789), Tringa solitaria Wilson, 1813, Calidris canutus (Linnaeus, 1758), Calidris fuscicollis (Vieillot, 1819), Calidris bairdii (Coues, 1861), Calidris melanotos (Vieillot, 1819), Calidris himantopus (Bonaparte, 1826), Tryngites subruficollis (Vieillot, 1819) and Phalaropus tricolor (Vieillot, 1819). There were also four Southern Austral Migrant species: Theristicus

Table 1. Non-passerine bird species recorded in Laguna Melincué Ramsar Site, Santa Fe province, Argentina. RA (relative abundance): VA = very abundant, $\mathrm{A}=$ abundant, $\mathrm{R}=$ rare, $\mathrm{S}=$ scarce; $\mathrm{MS}$ (migratory status): $\mathrm{R}=$ resident, $\mathrm{N}=$ Nearctic, NAM = northern austral migrants, SAM = southern austral migrants, Alt $=$ altitudinal migrants, and $\mathrm{P}=$ partial migrants. $\mathrm{N}=$ nesting. The systematic order follows Remsen et al. (2013).

\begin{tabular}{|c|c|c|c|c|}
\hline Family / species & English name & RA & MS & $\mathbf{N}$ \\
\hline \multicolumn{5}{|l|}{ Tinamidae } \\
\hline Nothura maculosa (Temminck, 1815) & Spotted Nothura & $\mathrm{S}$ & $\mathrm{R}$ & $\mathrm{N}$ \\
\hline \multicolumn{5}{|l|}{ Anhimidae } \\
\hline Chauna torquata (Oken, 1816) & Southern Screamer & $\mathrm{R}$ & $\mathrm{R}$ & $\mathrm{N}$ \\
\hline \multicolumn{5}{|l|}{ Anatidae } \\
\hline Dendrocygna bicolor (Vieillot, 1816) & Fulvous Whistling-duck & $\mathrm{S}$ & $\mathrm{P}$ & \\
\hline Dendrocygna viduata (Linnaeus, 1766) & White-faced Whistling-duck & A & $\mathrm{P}$ & \\
\hline Cygnus melancoryphus (Molina, 1782) & Black-necked Swan & $\mathrm{S}$ & $\mathrm{P}$ & \\
\hline Coscoroba coscoroba (Molina, 1782) & Coscoroba Swan & A & $P$ & $\mathrm{~N}$ \\
\hline Callonetta leucophrys (Vieillot, 1816) & Ringed Teal & $\mathrm{R}$ & $P$ & \\
\hline Amazonetta brasiliensis (Gmelin, 1789) & Brazilian Teal & $\mathrm{R}$ & $P$ & \\
\hline Anas sibilatrix Poeppig, 1829 & Chiloe Wigeon & $\mathrm{S}$ & $\mathrm{P}$ & \\
\hline Anas flavirostris Vieillot, 1816 & Yellow-billed Teal & A & $\mathrm{R}$ & $\mathrm{N}$ \\
\hline Anas georgica Gmelin, 1789 & Yellow-billed Pintail & A & $\mathrm{R}$ & $\mathrm{N}$ \\
\hline Anas bahamensis Linnaeus, 1758 & White-cheeked Pintail & A & $\mathrm{R}$ & $\mathrm{N}$ \\
\hline
\end{tabular}


Table 1. Continued.

\begin{tabular}{|c|c|c|c|c|}
\hline Family / species & English name & RA & MS & $\mathbf{N}$ \\
\hline Anas versicolor Vieillot, 1816 & Silver Teal & A & $\mathrm{R}$ & $\mathrm{N}$ \\
\hline Anas cyanoptera Vieillot, 1816 & Cinnamon Teal & $\mathrm{R}$ & $P$ & \\
\hline Anas platalea Vieillot, 1816 & Red Shoveler & VA & $\mathrm{R}$ & \\
\hline Netta peposaca (Vieillot, 1816) & Rosy-billed Pochard & $A$ & $P$ & \\
\hline Heteronetta atricapilla (Merrem, 1841) & Black-headed Duck & $\mathrm{S}$ & $P$ & \\
\hline Oxyura vittata (Philippi, 1860) & Lake Duck & $\mathrm{R}$ & $\mathrm{R}$ & \\
\hline \multicolumn{5}{|l|}{ Podicipedidae } \\
\hline Rollandia rolland (Quoy and Gaimard, 1824) & White-tufted Grebe & $A$ & $\mathrm{R}$ & \\
\hline Podilymbus podiceps (Linnaeus, 1758) & Pied-billed Grebe & $\mathrm{S}$ & $\mathrm{R}$ & \\
\hline Podiceps major (Boddaert, 1783) & Great Grebe & $\mathrm{S}$ & $\mathrm{R}$ & $\mathrm{N}$ \\
\hline Podiceps occipitalis Garnot, 1826 & Silvery Grebe & $\mathrm{R}$ & $\mathrm{R}$ & \\
\hline \multicolumn{5}{|l|}{ Phoenicopteridae } \\
\hline Phoenicopterus chilensis Molina, 1782 & Chilean Flamingo & VA & $\mathrm{R}$ & $\mathrm{N}$ \\
\hline Phoenicoparrus andinus (Philippi, 1854) & Andean Flamingo & A & Alt & \\
\hline Phoenicoparrus jamesi Sclater, 1886 & James's Flamingo & $\mathrm{R}$ & Alt & \\
\hline \multicolumn{5}{|l|}{ Ciconiidae } \\
\hline Ciconia maguari (Gmelin, 1789) & Maguari Stork & $\mathrm{S}$ & $\mathrm{R}$ & $\mathrm{N}$ \\
\hline Mycteria americana Linnaeus, 1758 & Wood Stork & $\mathrm{S}$ & NAM & \\
\hline \multicolumn{5}{|l|}{ Phalacrocoracidae } \\
\hline Phalacrocorax brasilianus (Gmelin, 1789) & Neotropic Cormorant & $A$ & $\mathrm{R}$ & \\
\hline \multicolumn{5}{|l|}{ Ardeidae } \\
\hline Ixobrychus involucris (Vieillot, 1823) & Stripe-backed Bittern & $\mathrm{R}$ & $\mathrm{R} ?$ & \\
\hline Nycticorax nycticorax (Linnaeus, 1758) & Black-crowned Night-heron & $\mathrm{S}$ & $\mathrm{R}$ & $\mathrm{N}$ \\
\hline Butorides striata (Linnaeus, 1758) & Striated Heron & $\mathrm{R}$ & $\mathrm{R}$ & \\
\hline Bubulcus ibis (Linnaeus, 1758) & Cattle Egret & $A$ & $\mathrm{R}$ & \\
\hline Ardea cocoi Linnaeus, 1766 & Cocoi Heron & $\mathrm{R}$ & $\mathrm{R}$ & \\
\hline Ardea alba Linnaeus, 1758 & Great Egret & $A$ & $\mathrm{R}$ & \\
\hline Syrigma sibilatrix (Temminck, 1824) & Whistling Heron & $\mathrm{S}$ & $\mathrm{R}$ & $\mathrm{N}$ \\
\hline Egretta thula (Molina, 1782) & Snowy Egret & A & $\mathrm{R}$ & \\
\hline \multicolumn{5}{|l|}{ Threskiornithidae } \\
\hline Plegadis chihi (Vieillot, 1817) & White-faced lbis & VA & $\mathrm{R}$ & \\
\hline Phimosus infuscatus (Lichtenstein, 1823) & Bare-faced Ibis & $\mathrm{S}$ & $\mathrm{R}$ & \\
\hline Theristicus melanopis (Gmelin, 1789) & Black-faced Ibis & $\mathrm{S}$ & SAM & \\
\hline Platalea ajaja Linnaeus, 1758 & Roseate Spoonbill & $A$ & NAM & \\
\hline Theristicus caerulescens (Vieillot, 1817) & Plumbeous Ibis & $\mathrm{R}$ & NAM & \\
\hline \multicolumn{5}{|l|}{ Accipitridae } \\
\hline Elanus leucurus (Vieillot, 1818) & White-tailed Kite & $\mathrm{S}$ & $\mathrm{R}$ & $\mathrm{N}$ \\
\hline Rostrhamus sociabilis (Vieillot, 1817) & Snail Kite & $\mathrm{S}$ & $\mathrm{R}$ & \\
\hline Circus cinereus Vieillot, 1816 & Cinereous Harrier & $\mathrm{R}$ & $\mathrm{R}$ & \\
\hline Circus buffoni (Gmelin, 1788) & Long-winged Harrier & $\mathrm{R}$ & $\mathrm{R}$ & \\
\hline Accipiter striatus Vieillot, 1808 & Sharp-shinned Hawk & $\mathrm{R}$ & $\mathrm{R} ?$ & \\
\hline Geranoaetus melanoleucus (Vieillot, 1819) & Black-chested Buzzard-eagle & $\mathrm{R}$ & $\mathrm{P}$ & \\
\hline Rupornis magnirostris (Gmelin, 1788) & Roadside Hawk & $\mathrm{S}$ & $\mathrm{R}$ & $\mathrm{N}$ \\
\hline Buteo swainsoni Bonaparte, 1838 & Swainson's Hawk & $\mathrm{R}$ & $\mathrm{N}$ & \\
\hline Geranoaetus albicaudatus (Vieillot, 1816) & White-tailed Hawk & $\mathrm{R}$ & $\mathrm{P}$ & \\
\hline \multicolumn{5}{|l|}{ Aramidae } \\
\hline Aramus guarauna (Linnaeus, 1766) & Limpkin & $\mathrm{R}$ & $\mathrm{R}$ & \\
\hline \multicolumn{5}{|l|}{ Rallidae } \\
\hline Pardirallus sanguinolentus (Swainson, 1838) & Plumbeous Rail & $\mathrm{R}$ & $\mathrm{R}$ & \\
\hline Gallinula galeata (Lichtenstein, 1818) & Common Gallinule & $\mathrm{R}$ & $\mathrm{R}$ & \\
\hline Gallinula melanops (Vieillot, 1819) & Spot-flanked Gallinule & $\mathrm{R}$ & $\mathrm{R}$ & \\
\hline Fulica leucoptera Vieillot, 1817 & White-winged Coot & VA & $\mathrm{R}$ & $\mathrm{N}$ \\
\hline Fulica armillata Vieillot, 1817 & Red-gartered Coot & $\mathrm{R}$ & $\mathrm{R}$ & $\mathrm{N}$ \\
\hline Fulica rufifrons Philippi and Landbeck, 1861 & Red-fronted Coot & $\mathrm{S}$ & $\mathrm{R}$ & \\
\hline \multicolumn{5}{|l|}{ Charadriidae } \\
\hline Vanellus chilensis (Molina, 1782) & Southern Lapwing & VA & $\mathrm{R}$ & $\mathrm{N}$ \\
\hline Pluvialis dominica (Müller, 1776) & American Golden-plover & $\mathrm{R}$ & $\mathrm{N}$ & \\
\hline Charadrius collaris Vieillot, 1818 & Collared Plover & $\mathrm{S}$ & $\mathrm{R}$ & \\
\hline Charadrius falklandicus Latham, 1790 & Two-banded Plover & $\mathrm{R}$ & SAM & \\
\hline Charadrius modestus Lichtenstein, 1823 & Rufous-chested Dotterel & $\mathrm{R}$ & SAM & \\
\hline Oreopholus ruficollis (Wagler, 1829) & Tawny-throated Dotterel & $\mathrm{R}$ & SAM & \\
\hline
\end{tabular}


Table 1. Continued.

\begin{tabular}{|c|c|c|c|c|}
\hline Family / species & English name & RA & MS & $\mathbf{N}$ \\
\hline \multicolumn{5}{|l|}{ Recurvirostridae } \\
\hline Himantopus mexicanus (Müller, 1776) & Black-necked Stilt & VA & $\mathrm{R}$ & $\mathrm{N}$ \\
\hline \multicolumn{5}{|l|}{ Scolopacidae } \\
\hline Limosa haemastica (Linnaeus, 1758) & Hudsonian Godwit & $\mathrm{R}$ & $\mathrm{N}$ & \\
\hline Bartramia longicauda (Bechstein, 1812) & Upland Sandpiper & $\mathrm{R}$ & $\mathrm{N}$ & \\
\hline Tringa melanoleuca (Gmelin, 1789) & Greater Yellowlegs & $S$ & $\mathrm{~N}$ & \\
\hline Tringa flavipes (Gmelin, 1789) & Lesser Yellowlegs & $\mathrm{S}$ & $\mathrm{N}$ & \\
\hline Tringa solitaria Wilson, 1813 & Solitary Sandpiper & $\mathrm{R}$ & $\mathrm{N}$ & \\
\hline Calidris fuscicollis (Vieillot, 1819) & White-rumped Sandpiper & A & $\mathrm{N}$ & \\
\hline Calidris bairdii (Coues, 1861) & Baird's Sandpiper & $S$ & $\mathrm{~N}$ & \\
\hline Calidris melanotos (Vieillot, 1819) & Pectoral Sandpiper & $S$ & $\mathrm{~N}$ & \\
\hline Calidris himantopus (Bonaparte, 1826) & Stilt Sandpiper & $S$ & $\mathrm{~N}$ & \\
\hline Phalaropus tricolor (Vieillot, 1819) & Wilson's Phalarope & $S$ & $\mathrm{~N}$ & \\
\hline Calidris canutus (Linnaeus, 1758) & Red Knot & $S$ & $\mathrm{~N}$ & \\
\hline Gallinago paraguaiae (Vieillot, 1816) & South American Snipe & $\mathrm{R}$ & $\mathrm{P}$ & \\
\hline Tryngites subruficollis (Vieillot, 1819) & Buff-breasted Sandpiper & $\mathrm{R}$ & $\mathrm{N}$ & \\
\hline \multicolumn{5}{|l|}{ Jacanidae } \\
\hline Jacana jacana (Linnaeus, 1766) & Wattled Jacana & $\mathrm{R}$ & $\mathrm{R}$ & \\
\hline \multicolumn{5}{|l|}{ Rostratulidae } \\
\hline Nycticryphes semicollaris (Vieillot, 1816) & South American Painted-snipe & $\mathrm{R}$ & $\mathrm{R}$ & \\
\hline \multicolumn{5}{|l|}{ Laridae } \\
\hline Chroicocephalus maculipennis (Lichtenstein, 1823) & Brown-hooded Gull & VA & $\mathrm{R}$ & $\mathrm{N}$ \\
\hline Chroicocephalus cirrocephalus (Vieillot, 1818) & Gray-hooded Gull & A & $\mathrm{R}$ & $\mathrm{N}$ \\
\hline Larus dominicanus Lichtenstein, 1823 & Kelp Gull & $\mathrm{S}$ & $\mathrm{R}$ & $\mathrm{N}$ \\
\hline Gelochelidon nilotica (Gmelin, 1789) & Gull-billed Tern & $S$ & $\mathrm{R}$ & $\mathrm{N}$ \\
\hline Sterna trudeaui Audubon, 1838 & Snowy-crowned Tern & $\mathrm{R}$ & $\mathrm{P}$ & \\
\hline Sternula superciliaris (Vieillot, 1819) & Yellow-billed Tern & $S$ & $P$ & \\
\hline \multicolumn{5}{|l|}{ Rynchopidae } \\
\hline Rynchops niger Linnaeus, 1758 & Black Skimmer & $\mathrm{R}$ & $\mathrm{P}$ & \\
\hline \multicolumn{5}{|l|}{ Columbidae } \\
\hline Columbina picui (Temminck, 1813) & Picui Ground-dove & A & $\mathrm{R}$ & $\mathrm{N}$ \\
\hline Columba livia Gmelin, 1789 & Rock Pigeon & A & $\mathrm{R}$ & $\mathrm{N}$ \\
\hline Patagioenas picazuro (Temminck, 1813) & Picazuro Pigeon & A & $\mathrm{R}$ & $\mathrm{N}$ \\
\hline Patagioenas maculosa (Temminck, 1813) & Spot-winged Pigeon & $S$ & $\mathrm{R}$ & $\mathrm{N}$ \\
\hline Zenaida auriculata (Des Murs, 1847) & Eared Dove & VA & $\mathrm{R}$ & $\mathrm{N}$ \\
\hline \multicolumn{5}{|l|}{ Cuculidae } \\
\hline Coccyzus melacoryphus Vieillot, 1817 & Dark-billed Cuckoo & $S$ & $\mathrm{R}$ & \\
\hline Guira guira (Gmelin, 1788) & Guira Cuckoo & A & $\mathrm{R}$ & $\mathrm{N}$ \\
\hline Tapera naevia (Linnaeus, 1766) & Striped Cuckoo & $\mathrm{R}$ & $\mathrm{R}$ & \\
\hline \multicolumn{5}{|l|}{ Tytonidae } \\
\hline Tyto alba (Scopoli, 1769) & Barn Owl & $S$ & $\mathrm{R}$ & $\mathrm{N}$ \\
\hline \multicolumn{5}{|l|}{ Strigidae } \\
\hline Megascops choliba (Vieillot, 1817) & Tropical Screech-owl & $\mathrm{S}$ & $\mathrm{R}$ & $\mathrm{N}$ \\
\hline Athene cunicularia (Molina, 1782) & Burrowing Owl & A & $\mathrm{R}$ & $\mathrm{N}$ \\
\hline Asio flammeus (Pontoppidan, 1763) & Short-eared Owl & $\mathrm{R}$ & $\mathrm{R}$ & \\
\hline \multicolumn{5}{|l|}{ Trochilidae } \\
\hline Chlorostilbon lucidus (Shaw 1812) & Glittering-bellied Emerald & $\mathrm{s}$ & $\mathrm{R}$ & \\
\hline \multicolumn{5}{|l|}{ Alcedinidae } \\
\hline Megaceryle torquata (Linnaeus, 1766) & Ringed Kingfisher & $\mathrm{R}$ & $\mathrm{R}$ & \\
\hline Chloroceryle americana (Gmelin, 1788) & Green Kingfisher & $\mathrm{R}$ & $\mathrm{R}$ & \\
\hline \multicolumn{5}{|l|}{ Picidae } \\
\hline Colaptes melanochloros (Gmelin, 1788) & Green-barred Woodpecker & $S$ & $\mathrm{R}$ & $\mathrm{N}$ \\
\hline Colaptes campestris (Vieillot, 1818) & Campo Flicker & A & $\mathrm{R}$ & $\mathrm{N}$ \\
\hline \multicolumn{5}{|l|}{ Falconidae } \\
\hline Caracara plancus (Miller, 1777) & Southern Caracara & $S$ & $\mathrm{R}$ & $\mathrm{N}$ \\
\hline Milvago chimango (Vieillot, 1816) & Chimango Caracara & $A$ & $\mathrm{R}$ & $\mathrm{N}$ \\
\hline Falco sparverius Linnaeus, 1758 & American Kestrel & $S$ & $\mathrm{R}$ & \\
\hline Falco femoralis Temminck, 1822 & Aplomado Falcon & $\mathrm{R}$ & $\mathrm{R}$ & \\
\hline Falco peregrinus Tunstall, 1771 & Peregrine Falcon & $\mathrm{R}$ & $P$ & \\
\hline \multicolumn{5}{|l|}{ Psittacidae } \\
\hline Myiopsitta monachus (Boddaert, 1783) & Monk Parakeet & $A$ & $\mathrm{R}$ & $\mathrm{N}$ \\
\hline
\end{tabular}


melanopis (Gmelin, 1789), Charadrius falklandicus Latham, 1790, Charadrius modestus Lichtenstein, 1823 and Oreopholus ruficollis (Wagler, 1829), and three Northern Austral Migrants: Theristicus caerulescens (Vieillot, 1817), Platalea ajaja Linnaeus, 1758 and Mycteria americana Linnaeus, 1758. We also recorded 17 Partial Migrant species: Dendrocygna bicolor (Vieillot, 1816), Dendrocygna viduata (Linnaeus, 1766), Cygnus melancoryphus (Molina, 1782), Coscoroba coscoroba (Molina, 1782), Callonetta leucophrys (Vieillot, 1816), Amazonetta brasiliensis (Gmelin, 1789), Anas sibilatrix Poeppig, 1829, Anas cyanoptera Vieillot, 1816, Netta peposaca (Vieillot, 1816), Heteronetta atricapilla (Merrem, 1841), Geranoaetus melanoleucus (Vieillot, 1819), Geranoaetus albicaudatus (Vieillot, 1816), Falco peregrinus Tunstall, 1771, Gallinago paraguaiae (Vieillot, 1816), Sterna trudeaui Audubon, 1838, Sternula superciliaris (Vieillot, 1819) and Rynchops niger Linnaeus, 1758. Finally, we recorded the presence of two altitudinal migrants: Phoenicoparrus andinus (Philippi, 1854) and Phoenicoparrus jamesi Sclater, 1886.

Most species were rare $(n=41)$ or scarce $(n=36)$ (Table 1). However, eight species were very abundant: Anas platalea Vieillot, 1816, Phoenicopterus chilensis Molina, 1782, Plegadis chihi (Vieillot, 1817), Fulica leucoptera Vieillot, 1817, Vanellus chilensis (Molina, 1782), Himantopus mexicanus (Statius Muller, 1776), Chroicocephalus maculipennis (Lichtenstein, 1823) and Zenaida auriculata (Des Murs, 1847).

Thirty-six species were recorded doing nesting activities or rearing chicks (Table 1). During the survey period we observed colonies of Larus dominicanus Lichtenstein, 1823 and Phoenicopterus chilensis. We recorded two nesting events of the latter species in summer 1999 and 2010 (about 400 and 150 nests, respectively; M. Romano, pers. obs.).

\section{DISCUSSION}

In a previous study of this Ramsar site based on ten years of waterbird surveys (1992-2002), we reported 74 species from 17 families (Romano et al. 2005). In the present checklist, updated with information from 12 more survey years, we add 35 species: four waterbird species from three families: Phoenicoparrus jamesi, Oreopholus ruficollis, Megaceryle torquata (Linnaeus, 1766) and Chloroceryle americana (Gmelin, 1788) and 31 non-passerine landbird species from nine families (Table 1). These 109 species represent almost half of the non-passerine birds reported by Fandiño and Giraudo (2010) for the whole of Santa Fe province (i.e., 231 species). These results confirm the importance of this wetland as a site of high environmental heterogeneity and biological diversity within a homogeneous and lowdiversity agricultural matrix (Di Giacomo et al. 2005; Romano et al. 2005; Giraudo et al. 2008; Romano et al. 2014).
The importance of long-term surveys of waterbirds is suggested by the record of 18 bird species at Melincué Ramsar site that were not reported for this area according to the species distribution maps shown in a recently published field guide to the birds of the Santa Fe province (de la Peña 2010). Among them, there were 13 species of waterbirds: Chauna torquata (Oken, 1816), Amazonetta brasiliensis, Phoenicoparrus jamesi, Mycteria americana, Theristicus caerulescens, Charadrius falklandicus, Charadrius modestus, Calidris canutus, Tryngites subruficollis, Nycticryphes semicollaris (Vieillot, 1816), Rynchops niger, Megaceryle torquata and Chloroceryle americana) and five landbird species, Accipiter striatus Vieillot, 1808, Geranoaetus albicaudatus, Falco peregrinus, Coccyzus melacoryphus Vieillot, 1817, and Megascops choliba (Vieillot, 1817).

All waterbird species recorded at our study site were also reported at Laguna Mar Chiquita (Córdoba province), a very large wetland located $350 \mathrm{~km}$ north of Laguna Melincué in the Chaco region (Torres and Michelutti 2006). In contrast, 13 waterbirds recorded at Melincué were not detected in a series of 314 surveys carried out in different seasons at 66 wetlands in the southeast Córdoba province from 2007-2010, less than $100 \mathrm{~km}$ west of our studyarea (Brandolin et al. 2011). Most of these waterbird species are migrants: Phoenicoparrus jamesi, Theristicus caerulescens, Theristicus melanopis, Charadrius modestus, Oreopholus ruficollis, Gallinago paraguaiae, Calidris canutus, Tryngites subruficollis, Amazonetta brasiliensis, Sternula superciliaris, whereas few others are residents: Jacana jacana (Linnaeus, 1766), Megaceryle torquata and Chloroceryle americana. The abundance of these species was very low at Laguna Melincué, thus they were probably recorded because of our extensive and prolonged surveys carried out at this wetland.

This study confirms the winter presence of Andean and James's flamingos in the study area. The Andean Flamingo is classified as Vulnerable by the IUCN Species Survival Commission, as well as listed in Appendix II of the Convention on International Trade in Endangered Species (CITES), Appendix I of the Convention of Migratory Species (CMS), and the US Endangered Species Act (IUCN 2013). In contrast, the James's Flamingo is classified as Near Threatened by IUCN, listed in Appendix II of CITES and in Appendices I and II of CMS (IUCN 2013). Moreover, both flamingo species are classified as En peligro (i.e., Endangered) for Argentina (López Lanús et al. 2008).

The Andean Flamingo was first recorded in Laguna Melincué several years ago (Romano et al. 2002), and its numbers have fluctuated throughout this time period (Romano et al. 2009; Derlindati et al. 2014; M. Romano pers. obs.). In some years this wetland hosted more than $30 \%$ of its known world population (Caziani et al. 2007; 
Romano et al. 2008), leading to its inclusion as a priority site in the High Andean Flamingo Wetland Network (Marconi and Sureda 2008). Andean Flamingos were also recorded at Laguna Mar Chiquita, $350 \mathrm{~km}$ further north in Córdoba province (Torres and Michelutti 2006; Caziani et al. 2007) and in several lowland wetlands of the Pampas located at the same latitude that Melincué (Romano et al. 2008; Brandolin and Ávalos 2010; Brandolin et al. 2011). In contrast, James's Flamingos were recently reported for the first time in Pampas wetlands at Melincué in 2011 and 2012 (Cruz et al. 2013), and recorded again in winter 2013 (M. Romano pers. obs.). They were also recorded at Laguna Mar Chiquita (Torres and Michelutti 2006; Caziani et al. 2007), but there is no other winter record for James's Flamingos at this latitude (Cruz et al. 2013).

We recorded 10 individuals of Theristicus caerulescens in 1993 and three others in 1999. Our records records are important because to our knowledge there are very few reports of this species in the Pampas region (Zotta 1935; Narosky and Di Giacomo 1993; de la Peña 1999; Romano et al. 2005). This ibis inhabits wetlands of the Chaco region located farther north from our study site (de la Peña 1999, 2010).

It is also important to highlight the presence of several individuals of Calidris canutus at our study site: 128 in April 1999 (autumn), 18 in February 2007 and five in February 2008 (summer). In Argentina, this summer-migrant is usually observed along seashores from Buenos Aires to Tierra del Fuego. At Melincué, the higher number of individuals recorded in autumn, emphasizes the potential importance of this inland site as a resting and feeding area for this shorebird species while migrating to the Northern Hemisphere (Blanco et al. 1992). To our knowledge, besides our study site (Romano et al. 2005), there is only one other inland record from Mar Chiquita Lake (Nores and Yzurieta 1979; Torres and Michelutti 2006).

Laguna Melincué is also an important site for the reproduction of several waterbird species. Besides our own observations, it has been cited as an important and frequent nesting site for one tern (Gelochelidon nilotica (Gmelin, 1789)), three gulls (Chroicocephalus maculipennis, Chroicocephalus cirrocephalus (Vieillot, 1818), Larus dominicanus), some anatids including Coscoroba coscoroba and Anas bahamensis (Gmelin, 1789), and Phoenicopterus chilensis (Narosky and Izurieta 1973; de la Peña 1992).

This large wetland is affected by multiple factors such as long-term cycles of hydrological dynamics (Romano et al. 2005; Guerra et al. 2015), climate change and anthropogenic impact (Marconi and Sureda 2008; Romano et al. 2014). Some of these factors are common to other large wetlands of the Chaco-Pampas region (Piovano et al. 2009), whereas others are particular for this site (e.g., water pumping; Romano et al. 2006). All these factors in turn may affect wildlife dynamics. For instance, in the last decades the density of bird species in the Pampas region has been affected by the expansion of agriculture (Filloy and Bellocq 2007; Medán et al. 2011; Gavier-Pizarro et al. 2012). As a consequence, many bird species have decreased their densities due to loss of their habitats (Bilenca et al. 2012), while others have increased their numbers due to a higher provision of food (Gavier-Pizarro et al. 2012). The latter is possibly the reason for the high densities at Laguna Melincue of Plegadis chihi, Chroicocephalus maculipennis and Zenaida auriculata. Therefore, research and monitoring programs on habitat and species conservation must be carried out over a relatively long time span, in order to detect numerous events, factors and interactions that could not be recorded or may be hidden in short-term research programs.

In conclusion, in this updated checklist we provide information about new records and biological aspects of some non-passerine bird species at Melincué Ramsar site. We consider that this baseline knowledge of non-passerine birds based on long-term surveys will be useful to assist future conservation studies in this highly threatened area.

\section{ACKNOWLEDGEMENTS}

We would like to thank the owners of Estancia San Carlos for allowing access to their property, and Santos Sánchez and his family for their kind hospitality during field work. We thank several students for their assistance in the field, and Wildlife Conservation Society, Woodland Park Zoo and RAMSAR Convention for financial support.

\section{LITERATURE CITED}

Barral, M.P. and N.O. Maceira. 2012. Land-use planning based on ecosystem service assessment: a case study in the southeast Pampas of Argentina. Agriculture, Ecosystems and Environment 154: $34-43$

Bilenca, D., M. Codesido, C. González Fischer, L. Pérez Carusi, E. Zufiaurre and A. Abba. 2012. Impactos de la transformación agropecuaria sobre la biodiversidad en la provincia de Buenos Aires. Revista del Museo Argentino de Ciencias Naturales 14: 189-198.

Blanco, D., H. Rodríguez Goñi and G. Pugnali. 1992. La importancia de Punta Rasa, Buenos Aires, en la migración del Chorlo Rojizo (Calidris canutus). El Hornero 13: 203-206.

Brandolin, P.G. and M.Á. Ávalos. 2010. Nuevos registros estivales de Flamenco Andino Phoenicoparrus andinus para la llanura central de Argentina, Provincia de Córdoba. Cotinga 32: 5-7.

Brandolin, P.G., M.Á. Ávalos and R. Martori. 2011. Waterbirds from wetlands of the southeast of the Córdoba province, Argentina. Check List 7(4): 537-541.

Brandolin, P.G., M.Á. Ávalos and C. de Angelo. 2013. The impact of flood control on the loss of wetlands in Argentina. Aquatic Conservation: Marine and Freshwater Ecosystems 23(2): 291-300. Canevari, P., D.E. Blanco, E.H. Bucher, G. Castro and I. Davidson. 1998. Los humedales de la Argentina. Clasificación, situación 
actual, conservación y legislación. Buenos Aires: Wetlands International. $208 \mathrm{pp}$.

Caziani, S.M., O. Rocha Olivio, E. Rodríguez Ramírez, M.C. Romano, E.J. Derlindati, A. Tálamo, D. Ricalde, C. Quiroga, J.P. Contreras, M. Valqui and H. Sosa. 2007. Seasonal distribution, abundance, and nesting of Puna, Andean, and Chilean flamingos. The Condor 109(2): 276-287.

Cruz, N.N., C. Barisón, M. Romano, F. Arengo, E.J. Derlindati and I. Barberis. 2013. A new record of James's flamingo (Phoenicoparrus jamesi) from Laguna Melincué, a lowland wetland in East-Central Argentina. The Wilson Journal of Ornithology 125(1): 217-221.

de la Peña, M.R. 1992. Guía de aves argentinas. Volume II. Santa Fe: L.O.L.A. (Literature of Latin America). $182 \mathrm{pp}$.

de la Peña, M.R. 1999. Aves argentinas. Lista y distribución. L.O.L.A. (Literature of Latin America). 244 pp.

de la Peña, M.R. 2010. Guía de aves de la provincia de Santa Fe. Santa Fe: Ediciones Grupo Cuenca. 463 pp.

Derlindati, E.J., M.C. Romano, N.N. Cruz, C. Barisón, F. Arengo and I.M. Barberis. 2014. Seasonal activity patterns and abundance of Andean flamingo (Phoenicoparrus andinus) at two contrasting wetlands in Argentina. Ornitología Neotropical 25(3): 317-331.

Di Giacomo, A.G., H. Luna, M. Manassero and L. Acquaviva. 2005. Laguna Melincué; pp. 439-440, In: A.S. Di Giacomo (ed.). Áreas importantes para la conservación de las aves en la Argentina. Buenos Aires: Aves Argentinas/Asociación Ornitológica del Plata.

Fandiño, B. and A.R. Giraudo. 2010. Revisión del inventario de aves de la provincia de Santa Fe, Argentina. Revista FABICIB 14: 116-137.

Filloy, J. and M.I. Bellocq. 2007. Respuesta de las aves rapaces al uso de la tierra: un enfoque regional. El Hornero 22(2): 131-140.

Gavier-Pizarro, G.I., N.C. Calamari, J.J. Thompson, S.B. Canavelli, L.M. Solari, J. Decarre, A.P. Goijman, R.P. Suárez, J.N. Bernardos and M.E. Zaccagnini. 2012. Expansion and intensification of row crop agriculture in the Pampas and Espinal of Argentina can reduce ecosystem service provision by changing avian density. Agriculture, Ecosystems and Environment 154: 44-55.

Giraudo, A., B. Fandiño, V. Arzamendia and G. Bellini. 2008. Aves nuevas o poco conocidas de Santa Fe (Argentina), incluyendo los Sitios Ramsar Jaaukanigás y Melincué. Natura Neotropicalis 39 89-99.

Guerra, L., E.L. Piovano, F.E. Córdoba, F. Sylvestre and S. Damatto. 2015. The hydrological and environmental evolution of shallow Lake Melincué, central Argentinean Pampas, during the last millennium. Journal of Hydrology 529(2): 570-583.

IUCN (International Union for the Conservation of Nature). 2013. IUCN Red List of threatened species. Version 2013.1. Accessed at http://www.iucnredlist.org/, 5 August 2013.

Laterra, P., M.E. Orúe and G.C. Booman. 2012. Spatial complexity and ecosystem services in rural landscapes. Agriculture, Ecosystems and Environment 154(1): 56-67.

López Lanús, B., P. Grilli, A.S. Di Giacomo, E.E. Coconier and R. Banchs. 2008. Categorización de las aves de la Argentina según su estado de conservación. Buenos Aires: Aves Argentinas /AOP and Secretaría de Ambiente y Desarrollo Sustentable. 64 pp.

Marconi, P.M. and A.L. Sureda. 2008. High Andean Flamingo Wetland Network: evaluation of degree of implementation of priority sites - preliminary results. Flamingo 16: 36-40.

Medán, D., J. Torretta, K. Hodara, E. de la Fuente and N. Montaldo. 2011. Effects of agriculture expansion and intensification on the vertebrate and invertebrate diversity in the Pampas of Argentina. Biodiversity and Conservation 20(13): 3077-3100.

Narosky, S. and D. Izurieta. 1973. Nidificación de la Gaviota de Cabeza Gris (Larus cirrhocephalus). El Hornero 11(3): 217-219.

Narosky, T. and A. G.Di Giacomo. 1993. Las aves de la provincia de
Buenos Aires. Distribución y estatus. Asociación Ornitológica del Plata, Vázquez Mazzini Editores, L.O.L.A. 128 pp.

Nores, M. and D. Yzurieta. 1979. Nuevas localidades para aves argentinas. Parte VIII. El Hornero 14: 45-52.

Piovano, E.L., D. Ariztegui, F. Córdoba, M. Cioccale and F. Sylvestre. 2009. Hydrological variability in South America below the Tropic of Capricorn (Pampas and Patagonia, Argentina) during the last 13.0 Ka; pp. 323-351, in: F. Vimeaux, F. Sylvestre and M. Khodri (eds.). Past climate variability in South America and surrounding regions. Dordrecht: Springer.

Remsen, J.V. Jr., C.D. Cadena, A. Jaramillo, M. Nores, J.F. Pacheco, M.B. Robbins, T.S. Schulenberg, F.G. Stiles, D.F. Stotz and K.J. Zimmer. 2013. A classification of the bird species of South America. American Ornithologists' Union. Accessed at http:// www.museum.lsu.edu/ Remsen/SACCBaseline.html, 20 August 2013.

Romano, M., F. Pagano and M. Luppi. 2002. Registros de Parina grande (Phoenicopterus andinus) en la laguna Melincué, Santa Fe, Argentina. Nuestras Aves 43: 15-17.

Romano, M.C., I.M. Barberis, F. Pagano and J.I. Maidagan. 2005. Seasonal and interannual variation in waterbird abundance and species composition in the Melincué saline lake, Argentina. European Journal of Wildlife Research 51(1): 1-13.

Romano, M. C., I. M. Barberis, F. Pagano and J. I. Maidagan. 2006. Laguna Melincué. Un humedal pampeano de importancia internacional. Nuestras Aves 51: 16-20.

Romano, M.C., I.M. Barberis, F. Pagano, P.M. Marconi and F. Arengo. 2008. Winter monitoring of Andean and Chilean Flamingos in lowland wetlands of central Argentina. Flamingo 16: 45-47.

Romano, M., I.M. Barberis, E. Derlindati, F. Pagano, P.M. Marconi and F. Arengo. 2009. Variation in abundance of Andean and Chilean Flamingos wintering in lowland wetlands of central Argentina in two contrasting years. Flamingo 17: 11-16.

Romano, M., I.M. Barberis, L. Guerra, E.L. Piovano and P. Minotti. 2014. Sitio Ramsar humedal Laguna Melincué: estado de situación. Santa Fe: Secretaría de Medio Ambiente de la Provincia de Santa Fe.

Saggese, M.D., R.P. Noseda, M.M. Uhart, S.L. Deem, H. Ferreyra, M.C. Romano, M.C. Ferreyra-Armas and M. Hugh-Jones. 2007. First detection of Bacillus anthracis in feces of free-ranging raptors from Central Argentina. Journal of Wildlife Diseases 43(1): 136-141.

The Ramsar Convention on Wetlands. 2013. The annotated Ramsar list of wetlands of international importance. Argentina. Accessed at http://www.ramsar.org/cda/en/ramsar-documents-list-annotatedramsar-17044/main/ramsar/1-31-218\%5E17044_40oo_o_.html, 20 August 2013.

Torres, R. and P. Michelutti. 2006. Aves acuáticas; pp. 237-249, in: E.H. Bucher (ed.). Bañados del Río Dulce y Laguna Mar Chiquita (Córdoba, Argentina). Córdoba: Academia Nacional de Ciencias.

Vega, E., G. Baldi, E.G. Jobbágy and J. Paruelo. 2009. Land use change patterns in the Río de la Plata grasslands: The influence of phytogeographic and political boundaries. Agriculture, Ecosystems and Environment 134(3-4): 287-292.

Viglizzo, E.F., F.C. Frank, L.V. Carreño, E.G. Jobbágy, H. Pereyra, J. Clatt, D. Pincén and M.F. Ricard. 2011. Ecological and environmental footprint of 50 years of agricultural expansion in Argentina. Global Change Biology 17(2): 959-973.

Zotta, A.R. 1935. Lista sistemática de las aves argentinas. El Hornero 6: $151-196$.

Received: 18 March 2014

Accepted: 7 October 2015

Academic editor: Thiago V. V. Costa 